Research Article

\title{
Cross-Spectral Analysis of Electrocardiographic and Nostril Airflow Signals Identifies Two Respiratory Frequencies of Heart Rate Modulation
}

\author{
Wan-An Lu $\mathbb{D}$, ${ }^{1}$ Jui-Feng Lin $\mathbb{D}^{2,3}$ Chen-Hsu Wang $\mathbb{D}^{2,}{ }^{4}$ Yung-Sheng Chen $\mathbb{D}^{,}{ }^{5}$ \\ Ying-Hua Shieh $\left(\mathbb{0},{ }^{6}\right.$ and Cheng-Deng Kuo $\oplus^{7,8,9}$ \\ ${ }^{1}$ Institute of Cultural Asset and Reinvention, Fo-Guang University, Yilan, Taiwan \\ ${ }^{2}$ Division of Neurosurgery, Department of Surgery, MacKay Memorial Hospital, Taipei, Taiwan \\ ${ }^{3}$ Institute of Traditional Medicine, National Yang-Ming University School of Medicine, Taipei, Taiwan \\ ${ }^{4}$ General Cardiology \& Coronary Care Unit, Cardiovascular Center, Cathay General Hospital, Taipei, Taiwan \\ ${ }^{5}$ Department of Exercise and Health Sciences, University of Taipei, Taipei, Taiwan \\ ${ }^{6}$ Department of Family Medicine, New Taipei Municipal Tucheng Hospital, New Taipei City, Taiwan \\ ${ }^{7}$ Department of Medical Research, Taipei Veterans General Hospital, Taipei, Taiwan \\ ${ }^{8}$ Department of Medicine, Taian Hospital, Taipei, Taiwan \\ ${ }^{9}$ Tanyu Research Laboratory, Taipei, Taiwan
}

Correspondence should be addressed to Cheng-Deng Kuo; cdkuo23@gmail.com

Received 7 November 2020; Revised 6 January 2021; Accepted 11 January 2021; Published 25 January 2021

Academic Editor: Saverio Maietta

Copyright (C) 2021 Wan-An Lu et al. This is an open access article distributed under the Creative Commons Attribution License, which permits unrestricted use, distribution, and reproduction in any medium, provided the original work is properly cited.

Respiration is known to be a significant modulator of heart rate, and the high-frequency component in the power spectrum of heart rate variability (HRV) is believed to be caused mainly by respiration. To investigate the effect of respiration on heart rate, cross-spectral analysis of electrocardiographic (ECG) and nostril airflow signals was performed in healthy subjects to find the common features of ECG and respiration. Forty-two healthy subjects were included in this study. The autospectra of respective ECG and nostril airflow signals and the cross-spectra of ECG and nostril airflow signals were obtained and compared with the corresponding conventional HRV measures. We found that there were two spectral peaks at around $0.03 \mathrm{~Hz}$ and $0.3 \mathrm{~Hz}$ in the autospectrum of nostril airflow and the cross-spectrum of ECG and nostril airflow. In addition, the cross-spectral normalized high-frequency power (nHFPcs) was significantly larger than that of conventional HRV, while the cross-spectral normalized very low-frequency power (nVLFPcs), normalized low-frequency power (nLFPcs), and low-/high-frequency power ratio (LHRcs) were significantly lower than those of the conventional HRV. The cross-spectral nLFPcs and LHRcs had positive correlations with their corresponding HRV measures. We conclude that cross-spectral analysis of ECG and nostril airflow signals identifies two respiratory frequencies at around $0.03 \mathrm{~Hz}$ and below and around $0.3 \mathrm{~Hz}$ and can yield significantly enhanced nHFPcs and significantly suppressed nVLFPcs, as compared to their counterparts in conventional HRV. Both very low-frequency and highfrequency components of HRV are caused in part or mainly by respiration.

\section{Introduction}

The rate and magnitude of heart beating are modulated by the sympathetic and parasympathetic branches of the autonomic nervous system. The variation in heart period due to the changes in the autonomic nervous controls and other physiological and pathological factors is termed heart rate variability (HRV). HRV analysis offers a noninvasive way to monitor and assess the autonomic nervous control of heart beating [1-3]. HRV analysis has been used in a variety of clinical settings to predict morbidity and mortality, to diagnose illness and to detect autonomic nervous dysfunction over a relatively short time period [4-6].

Respiration is one of the most important modulators of heart rate. The high-frequency component in HRV spectrum is known to be due to respiration $[7,8]$. In fact, HRV is also 
termed respiratory sinus arrhythmia, because heart rate oscillation can be influenced by the pattern and depth of breathing. The frequency at which the high-frequency component locates in the power spectrum of RR intervals is the respiratory frequency.

Power spectral analysis is often used to analyze the power or variance in the different frequency distributions of time series [9]. HRV analysis is often accomplished by performing fast Fourier transformation (FFT) of the time series of heart periods. Cross-spectral analysis is an extension of single Fourier spectral analysis to the simultaneous Fourier spectral analysis of two time series. It is often employed to uncover the correlations between two time series at different frequencies as a function of frequency and to find out the phase relationship between two time series. For instance, cross-spectral analysis of ECG and nostril airflow can be used to assess the cardiovascular-related functions of COPD patients [10].

Since respiration is one of the most important modulators of heart rate, examining the association between the electrocardiographic (ECG) and nostril airflow signals might give us more insight into the role played by respiration in the regulation of heart rate. The aims of this study were to investigate the effects of respiration on heart rate modulation and to compare the cross-spectral measures of ECG and nostril airflow with their corresponding conventional HRV measures.

\section{Subjects and Methods}

2.1. Study Subjects. Healthy subjects without cardiopulmonary disease and who were not using any medication were included in this study. All subjects were advised to not drink caffeinated beverages for at least 24 hours prior to ECG and nostril airflow signal recording. The Institutional Review Board of the Taipei Veterans General Hospital has approved this study (VGHTPE96-03-08A), and written informed consent was obtained from each subject before the study. This study was performed in accordance with the ethical standards laid down in the 1964 Declaration of Helsinki and its later amendments.

2.2. Data Collection. Forty-two healthy subjects were studied in supine position in an air-conditioned room with constant temperature and suitable humidity. After $5 \mathrm{~min}-$ utes' rest in supine position, a trend of lead II ECG signals and a trend of nostril airflow signals were recorded by a multichannel recorder (Biopac MP35, Biopac Systems, Inc., Goleta, CA, USA) for 12 minutes so that at least 512 heart periods can be obtained for HRV analysis and cross-spectral analysis. The recorded signals were transmitted to a laptop computer for storage and later offline analysis. During the period of recording, the patient was advised to close the eyes so as to minimize the interferences from the environment.

A hot-wire thermistor (SS6L temperature transducer, BIOPAC Systems Inc., Goleta, CA, USA) was placed directly underneath the nostril of the subject to record the airflow signals [10]. The operating principles for the hot-wire airflow measurement are thermal anemometry, which measures the total heat loss of a heating element and correlates the output signal to the flow rate of the fluid. When the air of breath flows through the sensor, the sensor measures the total heat loss. The temperature at the nostrils is inversely proportional to the airflow in and out of the nostril. The analogue voltage signal connected to the data acquisition instrument was then converted to digital signals and transmitted to a laptop computer for recording and later analysis.

2.3. HRV Analysis. The recorded ECG signals were retrieved afterwards to measure the consecutive RR intervals (RRIs) by using the software for the detection of $R$ wave. The analysis software was programmed using Mathcad 15 (Mathsoft Inc., Cambridge, MA, USA).

Both time and frequency domain HRV measures of 512 RRI were obtained for statistical and spectral analyses. The time domain measures such as mean RRI (mRRI), standard deviation $\left(\mathrm{SD}_{\mathrm{RR}}\right)$, coefficient of variation $\left(\mathrm{CV}_{\mathrm{RR}}\right)$, and root mean squared successive differences $\left(\mathrm{RMSSD}_{\mathrm{RR}}\right)$ of $512 \mathrm{RR}$ intervals were calculated using standard formulae. The power spectral density (PSD) of 512 RRI as a function of frequency was obtained by means of fast Fourier transformation (FFT) [11-14]. Direct current was excluded before the calculation of PSD. The area under the spectral peaks within the frequency range of $0.01-0.4 \mathrm{~Hz}, 0.01-0.04 \mathrm{~Hz}$, $0.04-0.15 \mathrm{~Hz}$, and $0.15-0.4 \mathrm{~Hz}$ were defined as the total power (TP), very low-frequency power (VLFP), low-frequency power (LFP), and high-frequency power (HFP), respectively. The normalized high-frequency power (nHFP $=$ HFP/TP) was used as the index of vagal modulation, the normalized low-frequency power $(\mathrm{nLFP}=\mathrm{LFP} / \mathrm{TP})$ as the index of combined sympathetic and vagal modulation, the low-/high-frequency power ratio $(\mathrm{LHR}=\mathrm{LFP} / \mathrm{HFP})$ as the index of sympathovagal balance [15], and the normalized very low-frequency power $(\mathrm{nVLFP}=\mathrm{VLFP} / \mathrm{TP})$ as the index of the renin-angiotensin-aldosterone system and vagal withdrawal [16].

2.4. Autospectra of ECG and Nostril Airflow Signals. A segment of ECG signals containing 512 RRI was used for spectral analysis (ECG autospectrum) by means of fast Fourier transformation. Similarly, a segment of nostril airflow signals of equal length to that of ECG signals was used for spectral analysis of airflow signals (nostril airflow autospectrum). Direct current was excluded before the calculation of the ECG and nostril airflow autospectra, respectively.

2.5. Cross-Spectral Analysis of ECG and Nostril Airflow Signals. Both ECG signals and nostril airflow signals with length equal to that of 512 RRI were Fourier-transformed to give the cross-spectrum of these two signals to assess the inter-relation between respiration and heart rate. The crossspectral density (CSD) of time series $x$ and $y$ as a function of frequency $f, \operatorname{CSD}_{x y}(f)$, is the Fourier transform of the crosscorrelation function (CCF) of time series $x$ and $y[10,17,18]$ : 


$$
\begin{gathered}
\operatorname{CCF}_{x y}(\tau)=\frac{1}{N} \sum_{k=0}^{N-k-1}\left(x_{k}-\bar{x}\right)\left(y_{k+\tau}-\bar{y}\right), \quad k=0, \ldots, N-1, \\
\operatorname{CSD}_{x y}(f)=\operatorname{FFT}\left[\operatorname{CCF}_{x y}(\tau)\right]=\frac{1}{N \Delta t} X(f) \cdot Y^{*}(f),
\end{gathered}
$$

where $\mathrm{CCF}_{x y}$ is the cross-correlation function of $x$ and $y, \bar{x}$ is the mean of $x, N$ is the sample size of the time series, FFT denotes fast Fourier transformation, $\Delta t$ is the sampling interval, $X(f)$ is the Fourier transform of $x$, and $Y^{*}(f)$ is the complex conjugate of $Y(f)$. The range of summation in the calculation of $\mathrm{CCF}_{x y}$ was limited to the range of available overlapped data of times series $x$ and $y$. The CSD displays the peaks at frequencies that are present in both signals being compared and can thus be used to detect the common rhythms in the two signals. The cross-spectral total power, very low-frequency power, low-frequency power, high-frequency power, normalized very low-frequency power, normalized low-frequency power, normalized high-frequency power, and low-/high-frequency power ratio were designated as TPcs, VLFPcs, LFPcs, HFPcs, nVLFPcs, nLFPcs, nHFPcs, and LHRcs, respectively, where the "cs" denoted "cross-spectral."

2.6. Statistical Analysis. Statistical analysis was performed to compare HRV measures with their corresponding crossspectral measures (SigmaPlot 13.0, SPSS Inc., Chicago, Illinois, USA). Since the absolute powers such as TP, VLFP, LFP, and HFP of HRV measures and the corresponding cross-spectral measures have different units, they cannot be compared statistically. Only the normalized powers of HRV and their corresponding normalized cross-spectral measures were compared.

The Wilcoxon signed rank test was used to compare the HRV measures and their corresponding cross-spectral measures. The correlations between HRV measures and their corresponding cross-spectral measures were analyzed using linear regression analysis. A $p<0.05$ was considered statistically significant.

\section{Results}

Table 1 shows the general characteristics of the study subjects. All data measured were within normal limits.

All data are presented as mean \pm SD. BMI, body mass index; SBP, systolic blood pressure; DBP, diastolic blood pressure; MABP, mean arterial blood pressure; HR, heart rate; bpm, beats per minute.

Figures 1(a) and 1(b) show the RRI tachogram and its HRV spectrum of a representative 54-year-old healthy male subject, respectively. The high-frequency component located at around $0.32 \mathrm{~Hz}$ corresponds to the respiratory frequency of the subject (Figure 1(b)). Figures 1(c) and 1(d) show the ECG waveform and its autospectrum of the same subject, respectively. The spectral peak located at around $1.0 \mathrm{~Hz}$ is the principal frequency or the heart rate (Figure 1(d)). Figures 1(e) and 1(f) show the nostril airflow waveform and its autospectrum of the same subject, respectively. The spectral peaks at around $0.3 \mathrm{~Hz}$ are
TABLE 1: Clinical characteristics of study subjects $(n=42)$.

\begin{tabular}{lc}
\hline Gender $(\mathrm{M} / \mathrm{F})$ & $36 / 6$ \\
Age $($ year $)$ & $47.8 \pm 19.9$ \\
Height $(\mathrm{cm})$ & $168.6 \pm 7.0$ \\
Weight $(\mathrm{kg})$ & $66.7 \pm 12.0$ \\
BMI $\left(\mathrm{kg} / \mathrm{m}^{2}\right)$ & $23.4 \pm 3.9$ \\
SBP $(\mathrm{mmHg})$ & $130.0 \pm 18.2$ \\
DBP $(\mathrm{mmHg})$ & $78.3 \pm 10.2$ \\
MABP $(\mathrm{mmHg})$ & $95.4 \pm 12.0$ \\
HR $(\mathrm{bpm})$ & $74.3 \pm 9.6$ \\
\hline
\end{tabular}

caused by respiration (Figure 1(f)). In addition, there are spectral peaks at around $0.03 \mathrm{~Hz}$ in the autospectrum of nostril airflow signals, indicating that $0.03 \mathrm{~Hz}$ is also one of the respiratory frequency of the subject. Figures $1(\mathrm{~g})$ and $1(\mathrm{~h})$ show the cross-correlation function between ECG and nostril airflow signals and the cross spectrum of ECG and nostril airflow, respectively. The spectral peaks located at around $0.3 \mathrm{~Hz}$ and $1.0 \mathrm{~Hz}$ correspond to the respiratory rate and heart rate, respectively (Figure 1(h)). In addition, there are again spectral peaks located at around $0.03 \mathrm{~Hz}$, suggesting that the frequency at around $0.03 \mathrm{~Hz}$ is also one of the respiratory frequency of the subject in addition to that around $0.3 \mathrm{~Hz}$.

Figure 2 compares the normalized HRV measures and the corresponding normalized cross-spectral measures of ECG and nostril airflow in healthy subjects. The crossspectral nHFPcs is greater than the nHFP of conventional HRV, while the nVLFPcs and LHRcs are smaller than the corresponding nVLFP and LHR of conventional HRV. It is interesting to note that the nVLFPcs is not zero, suggesting that the very low-frequency components do exist in both ECG and nostril airflow signals.

Correlation analysis shows that the nLFPcs and LHRcs had positive correlations with their corresponding conventional nLFP and LHR, respectively. There are no correlations between the nHFPcs and nHFP, or between HFPcs and HFP (Figure 3).

\section{Discussion}

The high-frequency component at about $0.3 \mathrm{~Hz}$ in the power spectrum of HRV is generally believed to be caused by respiration which is one of the most important modulators of heart rate. Cross-spectral analysis is known to be capable of extracting the common features of two signals, and to find the dependence of one signal over the other. This study used crossspectral analysis of these two signals to investigate the effect of respiration on heart rate. The autospectra of ECG and nostril airflow signals and the cross-spectra of ECG and nostril airflow signals were obtained for comparison. We found that there are two spectral peaks at around $0.03 \mathrm{~Hz}$ and below and at around $0.3 \mathrm{~Hz}$ in the autospectrum of nostril airflow and in the crossspectrum of ECG and nostril airflow. This finding suggested that there are two respiratory frequencies that are modulating the heart rate and that both very low-frequency component and the high-frequency component in the power spectrum of heart rate are related to respiration.

The very low-frequency component in the power spectrum of HRV is generally believed to be related to the renin-angiotensin-aldosterone system, vagal withdrawal, kidney 


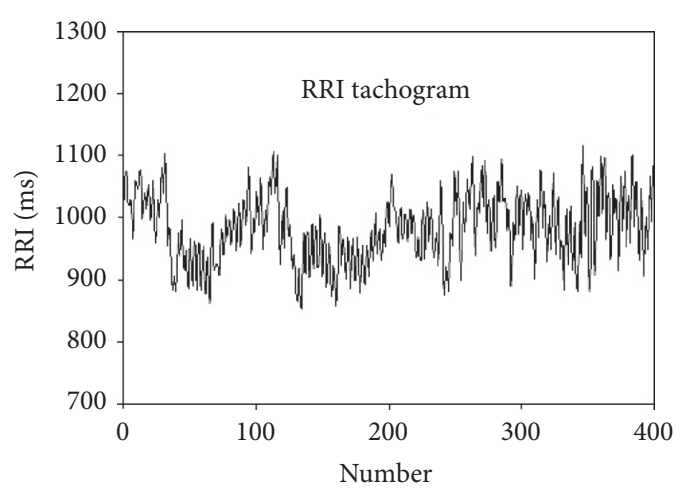

(a)

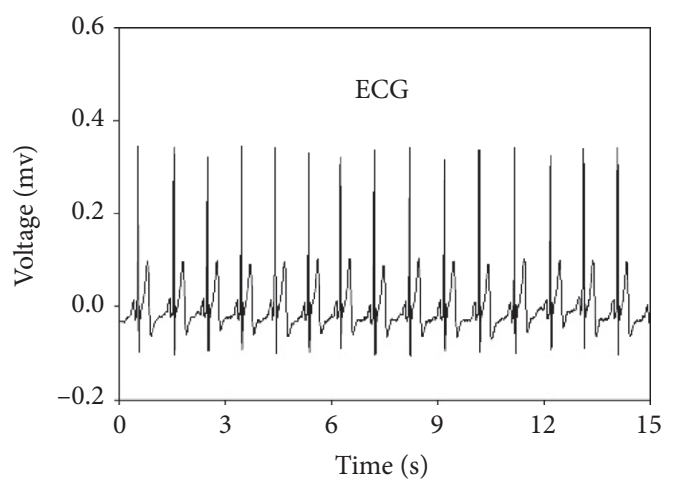

(c)

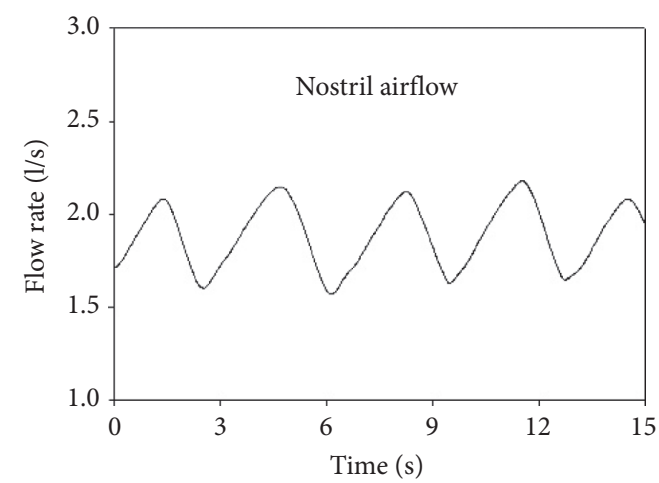

(e)

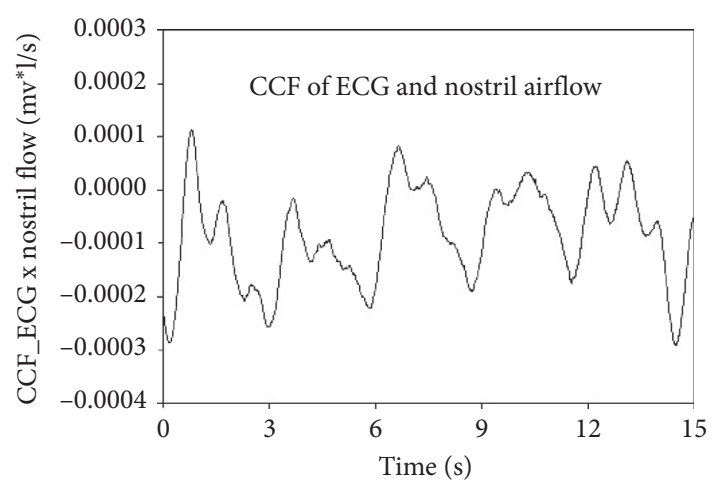

(g)

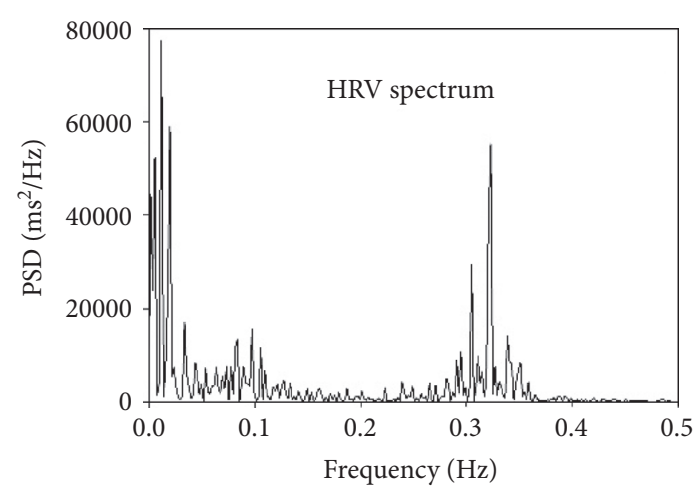

(b)

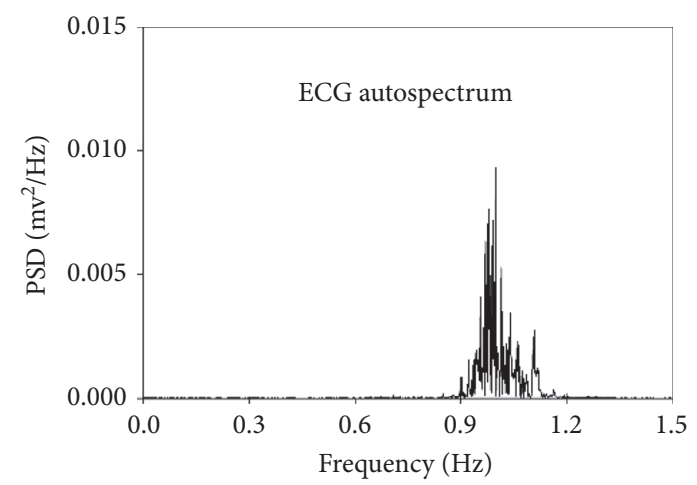

(d)

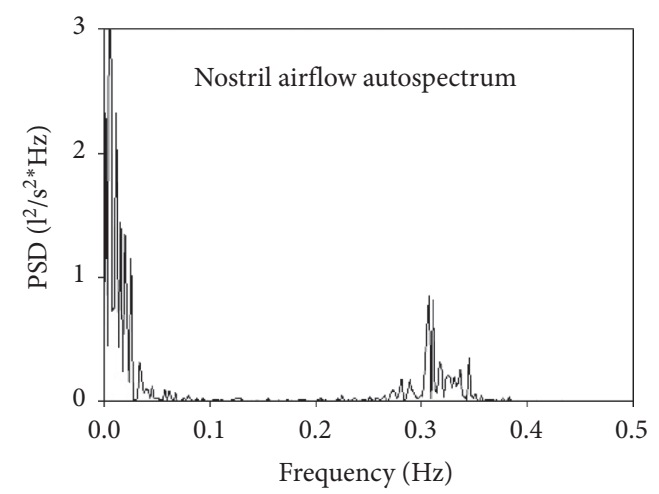

(f)

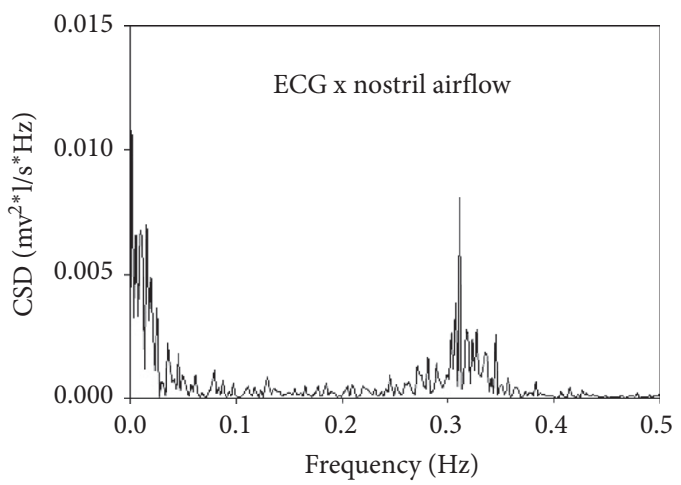

(h)

Figure 1: The RRI tachogram (panel (a)), HRV power spectrum (panel (b)), ECG waveform (panel (c)), ECG autospectrum (panel (d)), nostril airflow waveform (panel (e)), nostril airflow autospectrum (panel (f)), the autocorrelation function of ECG (panel (g)), and the crossspectrum of ECG and nostril airflow (panel (h)) of a representative subject. CCF = cross-correlation function, CSD = cross-spectral density, $\mathrm{ms}=$ millisecond, $\mathrm{s}=$ second, $\mathrm{mv}=$ millivolt, $\mathrm{l}=$ liter, and $\mathrm{Hz}=1 / \mathrm{s}$. 


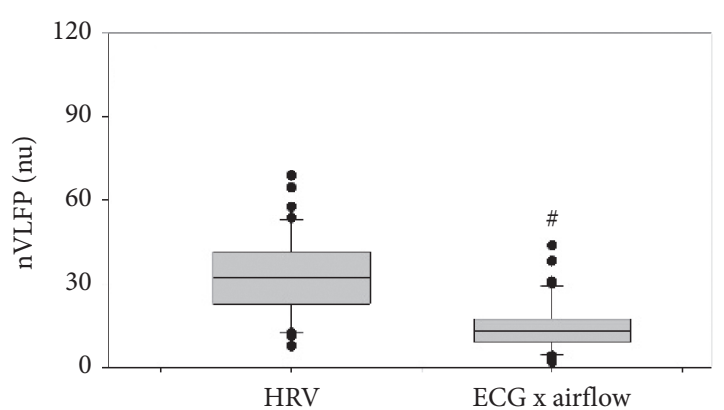

(a)

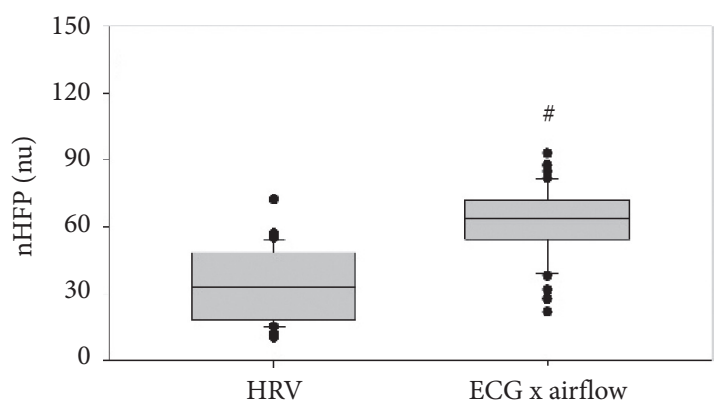

(c)

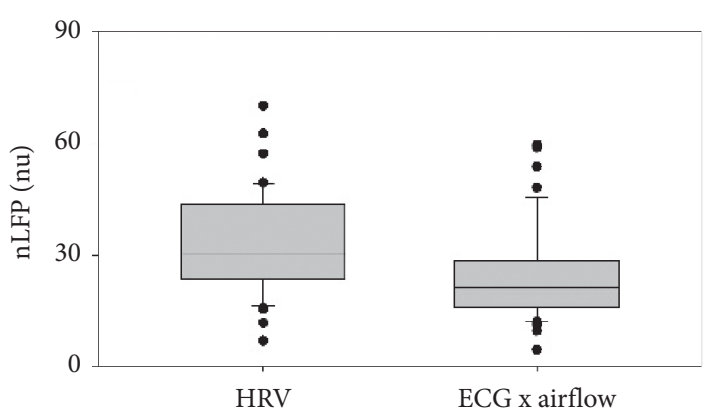

(b)

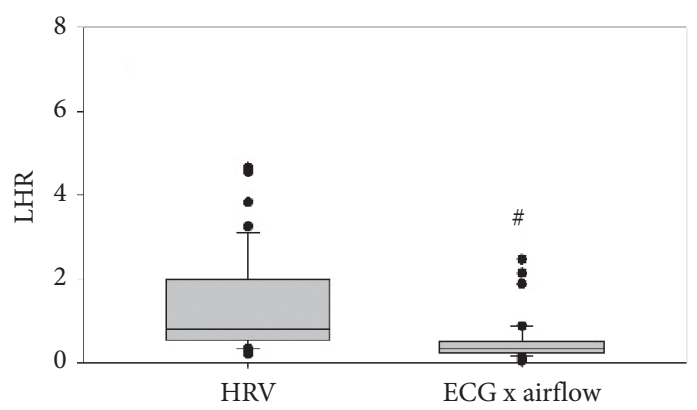

(d)

FIGURE 2: Comparison between measures of HRV and cross-spectral measures of ECG and nostril airflow. \#: $p<0.05$ vs. conventional HRV measures. $\mathrm{nu}=$ normalized unit.

function, and thermoregulation $[13,15,19]$. In this study, we found that respiration might also play a role in the regulation of heart rate in the very low-frequency region, because the spectral peaks at around $0.03 \mathrm{~Hz}$ and below can be found in both the autospectrum of nostril airflow and the cross-spectrum of ECG and nostril airflow (Figure 1). Moreover, we found that the cross-spectral analysis of ECG and nostril airflow signals can yield significantly enhanced cross-spectral normalized highfrequency power (nHFPcs) and significantly suppressed crossspectral normalized very low-frequency power (nVLFPcs) and cross-spectral low-/high-frequency power ratio (LHRcs), as compared with their counterparts in conventional HRV (Figure 2). These results suggested that both around $0.03 \mathrm{~Hz}$ and below and $0.3 \mathrm{~Hz}$ are respiratory frequencies of the subjects and that respiration is another modulator of the very low-frequency component of heart rate, in addition to the renin-angiotensinaldosterone system and other physiological systems. Though the spectral peaks at around $0.03 \mathrm{~Hz}$ and below in the autospectrum of ECG is not so prominent as compared to the spectral peaks of the first harmonic (heart rate), the presence of spectral peaks at around $0.03 \mathrm{~Hz}$ and below in the cross-spectrum of ECG and nostril airflow indicates that the same frequency component is also present in the ECG tracing.

There are several noninvasive methods of respiration monitoring that use biological signals, such as respiratory sinus arrhythmia (RSA), change in heart rate associated with breathing, QRS amplitude modulation, and change in amplitude of the ECG associated with breathing [20, 21]. Nostril airflow recording apparently is another kind of respiration monitoring method. Thus, nostril airflow can be used in the studies of respiration itself and the interaction between respiration and heart beating. It is comprehensible that the cross-spectral measures of ECG and nostril airflow signals are related to HRV measures, because respiration is well known to affect the rhythm and amplitude of ECG. For instances, the HR is accelerated during inspiration and slowed down during expiration. During inspiration, the apex of the heart is stretched towards the abdomen because of the filling of the lungs and the shifting down of the diaphragm. During expiration, the elevation of the diaphragm helps empty the lungs and compresses the apex of the heart towards the breast. Thus, respiration can modulate not only the heart rate, but also the amplitude of ECG signal by rotating the electric cardiac vector and modify the ECG signals thereof [22]. All these induced changes in ECG signals by respiration can be uncovered by cross-spectral analysis of ECG and nostril airflow signals. This may explain why the cross-spectral measures of ECG and nostril airflow signals are related to but not the same as the HRV measures.

The HRV measures are obtained by estimating the power spectral density of the RR intervals, and then the powers are calculated within different frequency ranges [23]. The unit of the power spectral density of RRI is millisecond squared per $\mathrm{Hz}$, and the unit of the absolute powers of HRV is millisecond squared. In the cross-spectral analysis of ECG and nostril airflow signals, the unit of cross-spectral density is millivolt $*$ liter/sec per $\mathrm{Hz}$, because the unit of the ECG signal is millivolt and the unit of the nostril airflow signal is $1 / \mathrm{sec}$, and the unit of the absolute power of cross-spectral measures is millivolt $*$ liter/sec. Because of the difference in units, it can be understood that HRV measures and cross-spectral measures of ECG are not the same physiological quantities. Hence, the cross-spectral measures of ECG and nostril 
ECG x Nostril airflow vs. HRV
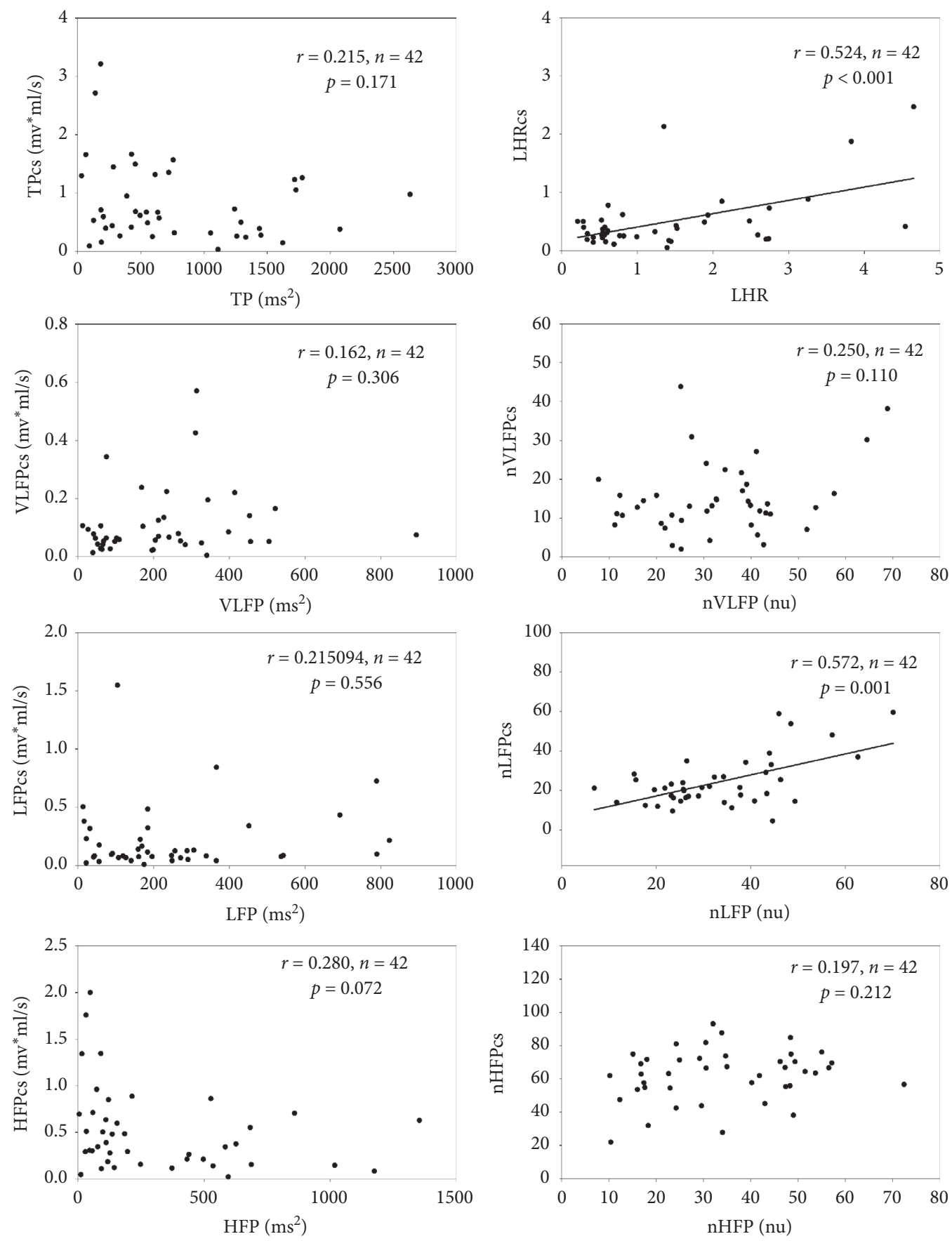

FIGURE 3: Linear regression analysis between HRV measures and corresponding cross-spectral measures of ECG and nostril airflow in healthy subjects. $\mathrm{cs}=$ cross-spectral, $\mathrm{nu}=$ normalized unit, and $\mathrm{ms}=$ millisecond.

airflow signals are related to but not equal to the HRV measures.

\section{Conclusions}

Cross-spectral analysis of ECG and nostril airflow signals can yield significantly magnified high-frequency component of HRV and significantly suppressed components other than the high-frequency component of HRV. Both
$0.03 \mathrm{~Hz}$ and below and around $0.3 \mathrm{~Hz}$ are the respiratory frequencies of the subject. Hence, both very low-frequency and high-frequency components of HRV are caused in part or mainly by respiration.

\section{Data Availability}

The data used to support the findings of this study are available from the corresponding author upon request. 


\section{Conflicts of Interest}

The authors declare no conflicts of interest.

\section{Acknowledgments}

This work was supported by the project TVGH-NTUH$96 \mathrm{VN}-010$ of Taipei Veterans General Hospital and National Taiwan University Hospital Joint Research Program.

\section{References}

[1] S. Akselrod, D. Gordon, F. Ubel, D. Shannon, A. Berger, and R. Cohen, "Power spectrum analysis of heart rate fluctuation: a quantitative probe of beat-to-beat cardiovascular control," Science, vol. 213, no. 4504, pp. 220-222, 1981.

[2] H. V. Huikuri, "Heart rate variability in coronary artery disease," Journal of Internal Medicine, vol. 237, no. 4, pp. 349-357, 1995.

[3] S. Yanagi, M. Yoshinaga, H. Horigome et al., "Heart rate variability and ambulatory blood pressure monitoring in young patients with hypertrophic cardiomyopathy," Circulation Journal, vol. 68, no. 8, pp. 757-762, 2004.

[4] J. T. Bigger, J. L. Fleiss, L. M. Rolnitzky, and R. C. Steinman, "The ability of several short-term measures of RR variability to predict mortality after myocardial infarction," Circulation, vol. 88, no. 3, pp. 927-934, 1993.

[5] R. E. Kleiger, P. K. Stein, and J. T. Bigger, "Heart rate variability: measurement and clinical utility," Annals of Noninvasive Electrocardiology, vol. 10, no. 1, pp. 88-101, 2005.

[6] F. Lombardi, G. Sandrone, S. Pernpruner et al., "Heart rate variability as an index of sympathovagal interaction after acute myocardial infarction," The American Journal of Cardiology, vol. 60, no. 16, pp. 1239-1245, 1987.

[7] T. E. Brown, L. A. Beightol, J. Koh, and D. L. Eckberg, "Important influence of respiration on human R-R interval power spectra is largely ignored," Journal of Applied Physiology, vol. 75, no. 5, pp. 2310-2317, 1993.

[8] J. A. Hirsch and B. Bishop, "Respiratory sinus arrhythmia in humans: how breathing pattern modulates heart rate," American Journal of Physiology-Heart and Circulatory Physiology, vol. 241, no. 4, pp. H620-H629, 1981.

[9] S. M. Kay and S. L. Marple, "Spectrum analysis-a modern perspective," Proceedings of the IEEE, vol. 69, no. 11, pp. 1380-1419, 1981.

[10] W.-A. Lu, J. Kuo, Y.-M. Wang et al., "Reduced enhancement of high-frequency component in the cross spectrum of ECG and nostril airflow signals in patients with chronic obstructive pulmonary disease," Physiological Reports, vol. 4, no. 7, p. e12763, 2016.

[11] S. Cerutti, M. Alberti, G. Baselli et al., "Automatic assessment of the interaction between respiration and heart rate variability signal," Medical Progress Through Technology, vol. 14, no. 1, pp. 7-19, 1988.

[12] A. M. Cerutti, L. T. Bianchi, and L. Mainardi, "Spectral analysis of the heart rate variability signal," in Heart Rate Variability, M. Malik and J. Camm, Eds., pp. 63-74, Futura Publishing Company, New York, NY, USA, 1995.

[13] Task Force of the European Society of Cardiology the North American Society of Pacing Electrophysiology, "Heart rate variability," Circulation, vol. 93, no. 5, pp. 1043-1065, 1996.

[14] B. Pomeranz, R. J. Macaulay, M. A. Caudill et al., "Assessment of autonomic function in humans by heart rate spectral analysis," American Journal of Physiology-Heart and Circulatory Physiology, vol. 248, no. 1, pp. H151-H153, 1985.

[15] M. Malik and A. J. Camm, Heart Rate Variability, Futura Publishing Company, Inc., New York, NY, USA, 1995.

[16] J. A. Taylor, D. L. Carr, C. W. Myers, and D. L. Eckberg, "Mechanisms underlying very-low-frequency RR-interval oscillations in humans," Circulation, vol. 98, no. 6, pp. 547-555, 1998.

[17] C. Chatfield, "Bivariate processes," in The Analysis of Time Series: An Introduction, pp. 136-147, Chapman \& Hall/CRC Press, New York, NY, USA, 6th edition, 2003.

[18] R. M. Rangayyan, Biomedical Signal Analysis: A Case-Study Approach, IEEE Press, Wiley-Interscience, John Wiley \& Sons, Inc., New York, NY, USA, 2002.

[19] G. G. Berntson, J. Thomas Bigger, D. L. Eckberg et al., "Heart rate variability: origins, methods, and interpretive caveats," Psychophysiology, vol. 34, no. 6, pp. 623-648, 1997.

[20] L. Mason, Signal processing methods for non invasive respiration monitoring, Ph.D. thesis, Trinity College, Oxford, UK, 2002.

[21] A. Travaglini, C. Lamberti, J. DeBie, and M. Ferri, "Respiratory signal derived from eight-lead ECG," Computers in Cardiology, vol. 25, pp. 65-68, 1998.

[22] G. Baselli, S. Cerutti, F. Badilini et al., "Model for the assessment of heart period and arterial pressure variability interactions and of respiration influences," Medical \& Biological Engineering \& Computing, vol. 32, no. 2, pp. 143-152, 1994.

[23] D. R. Brillinger, Time Series: Data Analysis and Theory, Holden Day, San Francisco, CA, USA, 2nd edition, 1981. 